\section{PO 8522 ESTABLISHING AN EQUITABLE GOVERNANCE FRAMEWORK FOR AN EBOLA DATA-SHARING PLATFORM}

Mahmoud Sama Cherif*, Elaine Craig, Samantha Strudwick, Alice Hawryszkiewycz, Laura Merson. Gamal Abdel Nasser University of Conakry, Guinea and Infectious Diseases Data Observatory, University of Oxford, UK

\subsection{6/bmjgh-2019-EDC.128}

Background Despite the potential public health gains of enabling access to patient-level data on emerging infections, the launch of a centralised, international platform to deliver on this has not been achieved to date. Barriers include: concerns over retention of national data ownership; patient privacy; appropriate consent; loss of academic recognition; criticism or exploitation of the data generators; perceived data misuse; and the challenges of sharing benefits with communities where data is generated.

Methods To determine the best approach to these issues in the context of Ebola, we have assembled a collaboration of partners including governments of Ebola-affected countries, non-government organisations, academic institutions, funders and public health authorities to form the Steering Committee of the Ebola Data Platform. Following stakeholder consultation, research and debate, the committee has developed a governance framework to enable access to emerging infections data, beginning with the data from the West African Ebola outbreak.

Results Promoting fair and equitable sharing of benefits that arise from the use of data is a key element of the framework. Strategies to secure this principle include integration of platform data management activities with the national health institutions in Ebola-affected countries and collaboration with research communities in these countries to determine research priorities and plan analyses. Public health benefit in affected countries is further supported via training and strengthening of research capacity and infrastructure.

Conclusion Developing a governance framework centered on the principle of equity has focused activities of the Ebola Data Platform on the affected health and research communities where they can have the most impact on patient outcomes, public health preparedness and future outbreak response.

\section{PO 8524 MOLECULAR CHARACTERISATION OF THE NOROVIRUS STRAINS RESPONSIBLE FOR ACUTE DIARRHOEA IN CHILDREN UNDER FIVE YEARS OLD HOSPITALISED IN BRAZZAVILLE}

${ }^{1}$ Vivaldie E Mikounou Louya*, ${ }^{1}$ Félix Koukouikila-Koussounda, ${ }^{2}$ Christew Vouvoungui, ${ }^{2}$ Simon Ch. Kobawila, ${ }^{3}$ Francine Ntoumi. ${ }^{1}$ Congolese Foundation for Medical Research, Brazzaville, Republic of the Congo; ${ }^{2}$ Faculty of Science and Technics, Marien Ngouabi University, Brazzaville, Republic of the Congo; ${ }^{3}$ Institute for Tropical Medicine, University of Tübingen, Tübingen, Germany

\subsection{6/bmjgh-2019-EDC.129}

Background Acute gastroenteritis is a leading cause of morbidity and mortality among children under five years old worldwide. Viruses are the most common responsible agent and norovirus is second after rotavirus. There is no published data on the occurrence of this agent in Brazzaville. This study aimed to determine the prevalence of norovirus infection and to evaluate the risk factors in hospitalised children in Brazzaville, Congo.

Methods From June 2012 to June 2013, stool samples were collected from children under five years old hospitalised with acute gastroenteritis at Makelekele hospital. Rotavirus and adenovirus infections were already characterised in this population. A total of 545 samples were tested for GI and GII norovirus infections using nested duplex reverse-transcriptionpolymerase chain reaction with specific primers. The positive samples will be sequenced and analysed to determine the corresponding genotype.

Results The GI and GII norovirus infection were found in 148 samples $(27,14 \%)$ in this study. Males $(28,85 \%)$ were more infected than females $(25 \%)$ but the difference was not significant. Norovirus infection was detected only in children under 24 months with a higher prevalence in the age group of $7-12$ months ( $p$ value $=0,048$ ). The norovirus infection was detected throughout the year, but it peaked during the dry season (August-September). Dual infection of rotavirus and norovirus was detected in 65 cases $(11,9 \%)$, rotavirus-adenovirus in 8 cases $(1,5 \%)$, norovirus-adenovirus in 4 cases $(0$, $73 \%)$. Triple infection was detected in 3 cases $(0,55 \%)$.

Conclusion This study suggests that norovirus infection is the second cause of gastroenteritis after rotavirus in the study area. However, further surveillance investigations need to be pursued in other sentinel sites of the country.

\section{PO 8527 GLUCOSE-6-PHOSPHATE DEHYDROGENASE DEFICIENCY AND ASSOCIATION WITH UNCOMPLICATED MALARIA IN CONGOLESE CHILDREN CONSULTING IN A PAEDIATRIC HOSPITAL IN BRAZZAVILLE}

${ }^{1}$ Nerly S Gampio Gueye*, ${ }^{2}$ Velavan P Thirumalaisamy, ${ }^{1}$ Christevy Vouvoungui, ${ }^{2}$ Simon Ch Kobawila, ${ }^{2}$ David Nderu, ${ }^{1} F$ élix Koukouikila-Koussounda, 'Francine Ntoumi. ${ }^{1}$ Congolese Foundation for Medical Research, Brazzaville, Republic of the Congo; ${ }^{2}$ Institute of Tropical Medicine, Universitätsklinikum Tübingen, Germany

\subsection{6/bmjgh-2019-EDC.130}

Background Malaria remains a public health problem in Republic of the Congo. The sub-microscopic infection including gametocytaemia constitutes a parasite reservoir that is recognised to contribute to malaria transmission. It is known that primaquine, an 8-aminoquinoline, is effective to eliminate Plasmodium falciparum (Pf) gametocytes. However, it induces haemolytic anaemia in individuals with glucose-6-phosphate dehydrogenase deficiency (G6PDd). It has been reported G6PDd also confers protection against severe malaria. To know the prevalence of G6PDd in the Congolese population is important in the case of future utilisation of this drug in the country. Therefore, in this study, we investigated 1) the prevalence of G6PDd in children infected with $P f$ and 2) the possible association between the presence of malaria, the presence of G6PD mutation and haemoglobin concentration.

Methods 229 children aged 1 to 10 years old presenting with fever (axillary $\mathrm{T}^{\circ} \geq 37.5^{\circ} \mathrm{C}$ ) were enrolled at the paediatric hospital Marien Ngouabi in Brazzaville. Thick and thin blood smears were done to detect and identify malaria parasites and determine parasite density. To detect the different glucose-6phosphate dehydrogenase genotypes, a $968 \mathrm{bp}$ fragment of the 